\title{
Rapid Deactivation of $\mathrm{N}\left({ }^{2} D\right)$ by O: Impact on Thermospheric and Mesospheric Odd Nitrogen
}

\author{
C. G. FESEN
}

Laboratory for Atmospheric and Space Physics, University of Colorado, Boulder, Colorado

J.-C. GÉRARD

Institut d'Astrophysique, Universite de Liege, Liège-Ougrée, Belgium

D. W. RUSCH

Laboratory for Atmospheric and Space Physics, University of Colorado, Boulder, Colorado

\begin{abstract}
One- and two-dimensional models of thermospheric odd nitrogen are used to explore the consequences of the recently measured fast quenching of $N\left({ }^{2} D\right)$ by $\mathrm{O}$. $A$ large rate coefficient for this reaction profoundly affects the odd-nitrogen chemistry by removing $N\left({ }^{2} D\right)$ as a source of NO and increasing the concentration of $N\left({ }^{4} S\right)$, which destroys NO. The model calculations show that, as the quenching rate increases, the NO and $N\left({ }^{2} D\right)$ densities decrease, while $N\left({ }^{4} S\right)$ densities increase. Comparisons with Atmosphere Explorer and Solar Mesosphere Explorer satellite observations are made. Use of the fast quenching rate in the models causes the NO peak altitude, typically observed near $110 \mathrm{~km}$, to rise to $140 \mathrm{~km}$. The $\mathrm{N}\left({ }^{2} \mathrm{D}\right)$ densities become 20 times smaller than those observed, while the modeled NO $\left(\mathrm{N}\left({ }^{4} S\right)\right.$ ) densities are roughly 2 to 3 times too small (large). Additional measurements of the $\mathrm{N}\left({ }^{2} D\right)+0$ quenching rate are clearly warranted. If the quenching rate is indeed very rapid, the chemistry of thermospheric odd nitrogen must be completely reexamined.
\end{abstract}

\section{INTRODUCTION}

The odd-nitrogen constituents NO, $N\left({ }^{4} S\right)$, and $N\left({ }^{2} D\right)$ are important in the chemistry and thermal structure of the thermosphere. The exothermic reactions involving the odd-nitrogen species are the dominant heat source from 150 to 250 $\mathrm{km}$ [Ridley et al., 1987]. NO also cools the thermosphere through infrared radiation at $5.3 \mu \mathrm{m}$, most effectively in the altitude region of $120-140 \mathrm{~km}$ [Ridley et al., 1987]. NO is particularly important in the radiative balance of this region during solar cycle maximum [Roble and Emery, 1983; Gérard and Roble, 1986, 1988]. Lower in the atmosphere, the ionization of NO by Lyman alpha radiation is important to the formation of the $D$ region [Nicolet and Aikin, 1960] while downward transport of thermospheric NO may perturb stratospheric ozone densities during polar nights [Solomon and $G$ arcia, 1984]. In the ionospheric $E$ region, NO controls the charge transfer rate from $\mathrm{O}_{2}^{+}$to $\mathrm{NO}^{+}$and thus the ionic composition.

Recently a serious complication has arisen in the NO chemistry. It has long been thought that the reaction of $\mathrm{N}\left({ }^{2} D\right)$ with $\mathrm{O}_{2}$ was the major source of $\mathrm{NO}$ in the thermosphere [Norton and Barth, 1970; Strobel et al., 1970; Rusch, 1973]. This assumption has come into serious question as a result of recent measurements of the deactivation of $\mathrm{N}\left({ }^{2} D\right)$ by O [Jusinski and Slanger, 1987; Jusinski et al., 1988]. A very

Copyright 1989 by the American Geophysical Union.

Paper number 88JA04208.

$0148-0227 / 89 / 88 \mathrm{JA}-04208 \$ 05.00$

high efficiency for this quenching process was measured, which essentially nullifies the reaction of $\mathrm{N}\left({ }^{2} D\right)$ and $\mathrm{O}_{2}$ as an effective source of NO. Consequently, the entire odd-nitrogen chemistry of the mesosphere and thermosphere must be reexamined. This paper utilizes both one- and two-dimensional odd-nitrogen models to investigate the consequences of fast $\mathrm{O}$ quenching of $\mathrm{N}\left({ }^{2} D\right)$ on the thermosphere. The model results are then compared to satellite measurements of $\mathrm{NO}$ and $\mathrm{N}\left({ }^{4} S\right)$, including observations obtained with the ultraviolet spectrometer on board the Solar Mesosphere Explorer satellite.

The theoretical description of the behavior of the odd-nitrogen species has been a challenge since nitric oxide was first observed in the thermosphere by Barth [1964]. Nitric oxide is an extremely variable constituent, with strong dependencies on latitude, season, solar cycle, and magnetic activity; longitudinal effects are also indicated [e.g., Stewart and Cravens, 1978; Cravens and Stewart, 1978; Cravens et al., 1985; Cravens and Killeen, 1988, and references therein]. The current theoretical understanding of NO chemistry as contained in most models is detailed here. The reactions and the adopted rate coefficients are given in Table 1.

The sources of thermospheric nitric oxide are predominantly

$$
\begin{aligned}
& \mathrm{N}\left({ }^{2} D\right)+\mathrm{O}_{2} \rightarrow \mathrm{NO}+\mathrm{O} \\
& \mathrm{N}\left({ }^{4} S\right)+\mathrm{O}_{2} \rightarrow \mathrm{NO}+\mathrm{O}
\end{aligned}
$$

while the loss is through 
TABLE 1. Important Reactions

\begin{tabular}{|c|c|c|c|}
\hline Number & Reaction & Rate & Reference \\
\hline $\begin{array}{l}\text { (1) } \\
(2) \\
(3) \\
(4) \\
(5) \\
(6) \\
(7) \\
(8) \\
(9) \\
(10)\end{array}$ & $\begin{array}{l}\mathrm{N}\left({ }^{2} D\right)+\mathrm{O}_{2} \rightarrow \mathrm{NO}+\mathrm{O} \\
\mathrm{N}\left({ }^{4} S\right)+\mathrm{O}_{2} \rightarrow \mathrm{NO}+\mathrm{O} \\
\left.\mathrm{N}^{4} S\right)+\mathrm{NO} \rightarrow \mathrm{N}_{2}+\mathrm{O} \\
\mathrm{NO}^{+}+e \rightarrow \mathrm{N}+\mathrm{O}^{+} \\
\mathrm{N}_{2}^{+}+\mathrm{O} \rightarrow \mathrm{N}\left({ }^{2} D\right)+\mathrm{NO}^{+} \\
\mathrm{N}_{2}+e \rightarrow \mathrm{N}+\mathrm{N}+e \\
\mathrm{~N}_{2}+\mathrm{hv} \rightarrow \mathrm{N}+\mathrm{N} \\
\left.\mathrm{N}^{2} D\right)+\mathrm{O} \rightarrow \mathrm{N}\left({ }^{4} S\right)+\mathrm{O} \\
\left.\mathrm{N}^{2} D\right)+e \rightarrow \mathrm{N}\left({ }^{4} S\right)+e \\
\left.\mathrm{~N}^{2} D\right) \rightarrow \mathrm{N}\left({ }^{4} S\right)+\mathrm{hv}\end{array}$ & $\begin{array}{l}5 \mathrm{E}-12^{+} \\
4.4 \mathrm{E}-12 \exp (-3220 / T) \\
3.4 \mathrm{E}-11 \\
4.2 \mathrm{E}-7\left(300 / T_{e}\right)^{0.85} \\
1.4 \mathrm{E}-10(\mathrm{~T} / 300)^{0.44} \\
\text { see reference } \\
\text { see reference } \\
\text { see text } \\
6.0 \mathrm{E}-10\left(T_{e}(300)^{0.5}\right. \\
1.07 \mathrm{E}-5 \mathrm{~s}\end{array}$ & $\begin{array}{l}\text { Lin and Kaufman [1971] } \\
\text { Becker et al. [1969] } \\
\text { Lee et al. [1978] } \\
\text { Kley et al. [1977] } \\
\text { McForland et al. [1974] } \\
\text { Strickdand and Meier [1982] } \\
\text { Richards et al. [1981] } \\
\text { Frederick and Rusch [1977] } \\
\text { Garstang [1956] }\end{array}$ \\
\hline
\end{tabular}

Units of $\mathrm{cm}^{3} \mathrm{~s}^{-1}$ unless otherwise indicated.

+ Read 5E-12 as $5 \times 10^{-12}$.

$$
\mathrm{N}\left({ }^{4} S\right)+\mathrm{NO} \rightarrow \mathrm{N}_{2}+\mathrm{O}
$$

Reaction (1) has been thought to dominate the NO production in the lower and middle thermosphere.

For $\mathrm{N}\left({ }^{2} D\right)$, the main sources are

$$
\begin{gathered}
\mathrm{NO}^{+}+e \rightarrow \mathrm{N}\left({ }^{2} D\right)+\mathrm{O} \\
\mathrm{N}_{2}^{+}+\mathrm{O} \rightarrow \mathrm{N}\left({ }^{2} D\right)+\mathrm{NO}^{+} \\
\mathrm{N}_{2}^{+}+e^{*} \rightarrow \mathrm{N}\left({ }^{2} D\right)+\left(\mathrm{N}\left({ }^{2} D\right), \mathrm{N}\left({ }^{4} S\right)\right)+e \\
\mathrm{~N}_{2}+\mathrm{hv} \rightarrow \mathrm{N}\left({ }^{2} D\right)+\mathrm{N}\left({ }^{4} S\right)
\end{gathered}
$$

while the loss is through

$$
\begin{gathered}
\mathrm{N}\left({ }^{2} D\right)+\mathrm{O}_{2} \rightarrow \mathrm{NO}+\mathrm{O} \\
\mathrm{N}\left({ }^{2} D\right)+\mathrm{O} \rightarrow \mathrm{N}\left({ }^{4} S\right)+\mathrm{O} \\
\mathrm{N}\left({ }^{2} D\right)+e \rightarrow \mathrm{N}\left({ }^{4} S\right)+e \\
\mathrm{~N}\left({ }^{2} D\right) \rightarrow \mathrm{N}\left({ }^{4} S\right)+\mathrm{hv}(520-\mathrm{nm})
\end{gathered}
$$

The assumption that reaction (1) provides the main source for $\mathrm{NO}$ is questionable if the removal of $\mathrm{N}\left({ }^{2} D\right)$ through reaction (9) is fast compared to the rate of reaction (1). Two sets of laboratory values now exist for the deactivation coefficient of $\mathrm{N}\left({ }^{2} D\right)$ by atomic nitrogen. Davenport et al. [1976] reported a value of $1.8 \pm 0.6 \times 10^{-12} \mathrm{~cm}^{-3} \mathrm{~s}^{-1}$ at 315 $\mathrm{K}$ whereas Iannuzzi and Kaufman [1980] obtained $1.8 \pm 0.3 \times$ $10^{-12} \mathrm{~cm}^{-3} \mathrm{~s}^{-1}$ at $300 \mathrm{~K}$. More recently, the temperature dependence has been measured [Jusinski and Slanger, 1987; Jusinski et al., 1988], and the coefficient was found to be 3.4 x $10^{-11} \exp \left(-145 / T_{n}\right)$. Consequently, the laboratory quenching rate is now measured to be at least 50 times as large as the rate determined by analysis of atmospheric measurements as described below. The effect of a large quenching rate is to remove $\mathrm{N}\left({ }^{2} D\right)$ as the dominant source of NO through reaction (1); this calls into question a number of other measurements and calculations involving each of the odd-nitrogen species.

In a series of papers using AE measurements of the 520-nm emission from $\mathrm{N}\left({ }^{2} D\right)$ and supporting data, Rusch et al. [1975], Frederick and Rusch [1977], and Rusch and Gérard [1980] each concluded that the quenching rate of $N\left({ }^{2} D\right)$ by $O$ had to be small (about $4 \times 10^{-13} \mathrm{~cm}^{3} \mathrm{~s}^{-1}$ ). Cogger et al. [1980] reached the same conclusion by combining Arecibo radar data with groundbased photometer observations. However, the rate determined in the laboratory is $2-3 \times 10^{-11}$ $\mathrm{cm}^{3} \mathrm{~s}^{-1}$, depending on temperature, or more than 50 times that which stems from the atmospheric determinations. The analysis of Frederick and Rusch [1977] showed that even if reactions (4)-(7) were $100 \%$ efficient in the production of $\mathrm{N}\left({ }^{2} D\right)$, a value for the $\mathrm{N}\left({ }^{2} D\right)+\mathrm{O}$ quenching rate 0.1 times the laboratory value could not satisfy the $520-\mathrm{nm}$ measurement. If the new laboratory value were adopted, no $520-\mathrm{nm}$ emission would have been detected by the Visible Airglow Experiment (VAE) [Hays et al., 1973] on AE. The maximum volume emission rate would have been less than 1 photon $\mathrm{cm}^{-3}$ $\mathrm{s}^{-1}$, far below the detection threshold. Additional analysis in the same paper of $N\left({ }^{2} D\right)$ nighttime emission gave the same low value for the quenching. At night, only one source is operative for $N\left({ }^{2} D\right)$ (reaction (4)). Even under the assumption of unit efficiency for $N\left({ }^{2} D\right)$ production from reaction (4), the data are not consistent with a large quenching rate. In an analysis of auroral $520-\mathrm{nm}$ emission, Rusch and Gérard [1980] reported vertical column brightness measurements of 15-30 rayleighs above about $150 \mathrm{~km}$. The magnitude of the emission, again, is consistent with a low quenching rate, provided the chemical scheme outlined above is valid.

A potential, although unlikely, solution to the discrepancy is that the radiative lifetime of the $N\left({ }^{2} D\right)$ state adopted in these studies is incorrect. If the radiative lifetime were significantly shorter than the 24 hours initially calculated by Garstang [1956] and confirmed by recent detailed computations [Godefroid and Froese Fischer, 1984; Zeippen, 1982], then the emission rate could be greater even if the state were rapidly quenched. For the magnitude of the emission to be near that measured by VAE, the radiative lifetime would have to decrease, to first order, by the ratio of the quenching rates determined in the atmosphere to that determined in the laboratory, or a factor of 50 . The required lifetime, under the assumption of fast quenching, to produce a volume emission rate near that measured would be about 0.5 hour. However, the assumed short radiative lifetime of $\mathrm{N}\left({ }^{2} D\right)$ does not alleviate the reduced production of NO through reaction (1.)

Two additional factors may enter into the theoretical picture. First, since several of the relevant reactions in the oddnitrogen chemical scheme have temperature-dependent rate coefficients, the computed number densities are quite sensitive to the temperature profile. Second, an intriguing idea 
involving the possibility of a population of translationally excited $N\left({ }^{4} S\right)$ atoms was suggested by Solomon [1983]. Some of these "hot" $N\left({ }^{4} S\right)$ atoms would preferentially react with $\mathrm{O}_{2}$ to produce NO instead of destroying NO through reaction (3.) Thus this process may constitute an additional source of $N$, but a quantitative evaluation of its efficiency requires additional information about hot atom chemistry.

\section{MODEL DESCRIPTIONS}

The dependence of the odd-nitrogen system on the $\mathrm{N}\left({ }^{2} D\right)$ quenching rate is investigated through use of one-dimensional and two-dimensional models. The one-dimensional model is time-dependent and basically similar to that described by Gerard and Taieb [1986]. The UV solar fluxes and their dependence on solar activity are taken from Torr and Torr [1985] with the corrections to the extreme ultraviolet fluxes suggested by Richards and Torr [1988]. The latitude-dependent eddy diffusion coefficients derived by Ebel [1980] for solstice conditions are adopted. The branching ratios for production of $\mathrm{N}\left({ }^{2} D\right)$ atoms by processes (6) and (7) are set to 0.6 and 0.5 , respectively. The quenching rate of $\left.\mathrm{N}^{2} D\right)$ by atomic oxygen is varied to investigate its effect on the calculated odd nitrogen species in comparison with observations.

The two-dimensional model is that of Gerard and Roble [1986, 1988], based on earlier modeling work by Roble and Kasting [1984] and Gérard et al. [1984]. This is a coupled chemical-dynamical-radiative model of the thermosphere which includes in full detail the chemical production and loss of $\mathrm{NO}, \mathrm{N}\left({ }^{4} S\right)$, and $\mathrm{N}\left({ }^{2} D\right)$, horizontal and vertical transport, and the effect of particle-induced ionization and heating in the auroral zones.

In brief, the model solves the zonal and meridional momentum, thermodynamic, continuity, hydrostatic, and diffusion equations for the mass mixing ratios of the major constituents, thereby calculating the time-dependent, twodimensional distribution of the $\mathrm{O}, \mathrm{O}_{2}$ and $\mathrm{N}_{2}$ densities and the neutral winds, pressure, and perturbation temperatures. Particle precipitation and a high-latitude heat source are included in the formulation, as is the infrared emission of NO in the $5.3-\mu \mathrm{m}$ band. The calculated zonal mean atmosphere is used to solve for the distribution of the minor neutral constituents $\mathrm{NO}, \mathrm{N}\left({ }^{4} S\right)$, and $\mathrm{N}\left({ }^{2} D\right)$ by updating the production and loss terms and the wind components in the continuity equations for the minor constituents at each time step. The calculated NO density is then used to update the net heating term in the thermodynamic equation for the mean atmosphere at the next time step. Ion densities are also computed assuming photochemical equilibrium. The latitudinal grid in the model extends from pole to pole in $5^{\circ}$ increments; the altitude scale is from 70 to $356 \mathrm{~km}$, in pseudo-log pressure coordinates, with variable step sizes which are approximately onethird the local atmospheric scale height.

Recent updates to the model include a seven-wavelengthinterval representation of the solar UV fluxes and photoionization and absorption cross sections of $\mathrm{O}, \mathrm{O}_{2}$, and $\mathrm{N}_{2}$ (R.G. Roble and B.A. Emery, private communication, 1983); updating of the ion drag coefficients at each time step; and addition of the heating due to the exothermic reaction of $\mathrm{N}+\mathrm{NO}$. Reaction rates and branching ratios were also updated where necessary to agree with more recent laboratory and theoretical developments. The branching ratios adopted for $\mathrm{N}\left({ }^{2} D\right)$ production by reactions (6) and (7) are 0.6 and 0.5 , respectively.
The two-dimensional model calculations correspond to northem summer solstice, solar cycle minimum conditions, with an $F 10.7$ index of 80 . The model calculations of Gerard and Roble [1986], which represent these same conditions, were used as initial conditions for the model runs. Thus, the neutral winds, temperatures, and densities of $\mathrm{O}, \mathrm{O}_{2}, \mathrm{~N}_{2}$, and of NO, $\mathrm{N}\left({ }^{4} S\right)$, and $\mathrm{N}\left({ }^{2} D\right)$ calculated by the Gerard and Roble [1986] study were used to start up the model. The numerical integration is run for $\mathbf{3 0}$ days, after which a quasi-steady state is reached.

\section{COMPARISONS OF MODEL TO DATA}

Model simulations were carried out for three values of the quenching rate of $N\left({ }^{2} D\right)$ by $O$, corresponding to high, intermediate, and low efficiency for the reaction. The reaction rates are $k_{0}=5 \times 10^{-13}, 1 \times 10^{-12}$, and $3.4 \times 10^{-11} \exp (-145 /$ $T n) \mathrm{cm}^{-3} \mathrm{~s}^{-1}$; these correspond, respectively, to the values deduced by Frederick and Rusch [1977] and Torr et al. [1976], based on $\mathrm{N}\left({ }^{2} D\right)$ airglow measurements, and the rate recently measured in the laboratory by Jusinski and Slanger [1987] and Jusinski et al. [1988].

\section{One-Dimensional Model Results}

The Atmosphere Explorer (AE) satellite series [Dalgarno et al., 1973] is particularly valuable in model validation since (nearly) simultaneous observations of the major ionic and neutral species were obtained. Richards et al. [1981] present AE-C altitude profiles of NO, $N\left({ }^{4} S\right)$, and $N\left({ }^{2} D\right)$ from February $14,1974,1400 \mathrm{LT}, 40^{\circ} \mathrm{N}$. The measurements were obtained by the Ultraviolet Nitric Oxide Experiment (UVNO) [Barth et al., 1973], Neutral Atmosphere Composition Experiment (NACE) [Spencer et al., 1973], and the Visible Airglow Experiment (VAE) [Hays et al., 1973] instruments, respectively. Figure 1 reproduces these $\mathrm{AE}$ measurements, along with the one-dimensional model results for similar conditions using the low, medium, and high quenching rates.

The model with the low quenching rate (Figure 1a) reproduces the $\mathrm{NO}$ and $\mathrm{N}\left({ }^{4} S\right)$ profiles fairly well, although the calculated NO $\left(\mathrm{N}\left({ }^{4} S\right)\right)$ densities are slightly larger (smaller) than observed. The calculated $\mathrm{N}\left({ }^{2} D\right)$ densities are also somewhat larger than observed. The calculations done with the medium quenching rate (Figure $1 b$ ) agree well with all three observed quantities. Model calculations with the fast quenching rate (Figure $1 c)$ produce $\mathrm{N}\left({ }^{2} D\right)$ densities that are completely unacceptable: they are roughly 20 times smaller than those observed. The NO densities are about 3 times smaller than observed, while the $\mathrm{N}\left({ }^{4} S\right)$ densities are overestimated by up to a factor of 2. The altitude of the peak NO density rises to around $140 \mathrm{~km}$ with the fast quenching rate, a location in disagreement with rocket and satellite observations [McCoy, 1983a,b; Cravens et al., 1985; Swider, 1978; Ogawa et al., 1984; Iwagami and Ogawa, 1987].

Model calculations were also obtained for solar cycle maximum conditions. The simulations exhibit the same general features as those for solar cycle minimum: as the quenching rate is increased, the lower thermosphere NO densities decrease, while the altitude of the peak rises, and the $\mathrm{N}\left({ }^{4} S\right)$ densities increase, with a pronounced bulge near $100 \mathrm{~km}$. The $\mathrm{N}\left({ }^{2} D\right)$ densities decrease to very small values; calculations with the large quenching rate are slightly greater than $10^{4} \mathrm{~cm}^{-3}$, which is less than for the solar cycle minimum case (Figure 1c). As for the solar cycle minimum case, the 

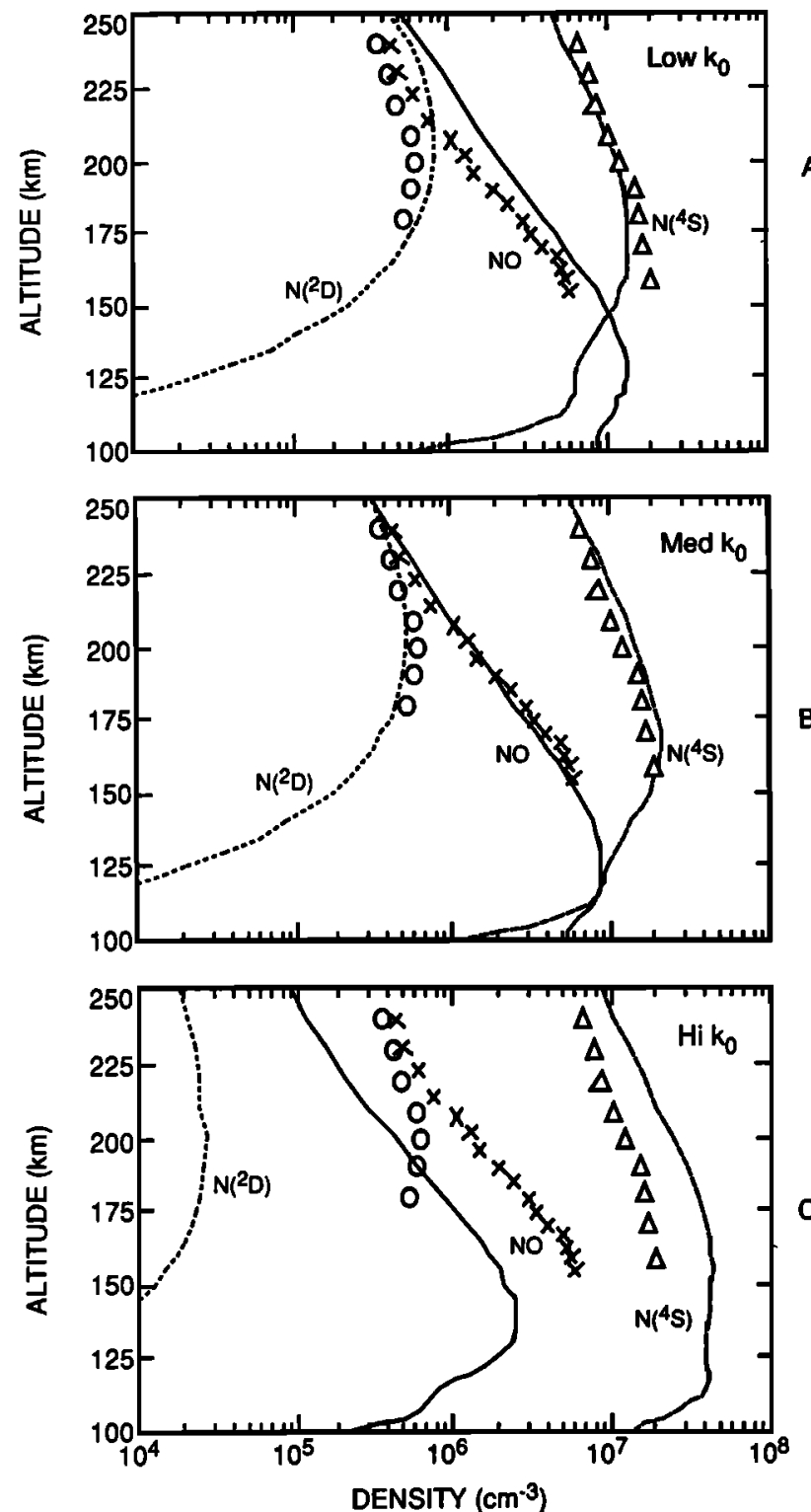

Fig. 1. Comparison of time-dependent one-dimensional model calculations (solid curves) with AE satellite observations (symbols) from Richards et al. [1981]. Observations made on day 46 of 1974, at $40^{\circ} \mathrm{N}, 1400 \mathrm{LT}$. Calculations made with different values of $\left.\mathrm{N}^{2} D\right)+\mathrm{O}$ quenching rate: (a) low quenching rate of $5.0 \times 10^{-13} \mathrm{~cm}^{3} \mathrm{~s}^{-1} ;(b)$ medium quenching rate of $1.0 \times 10^{-12} \mathrm{~cm}^{-3} \mathrm{~s}^{-1}$; and (c) fast quenching rate of $3.4 \times 10^{-11} \exp (-145 / \mathrm{Tn}) \mathrm{cm}^{-3} \mathrm{~s}^{-1}$.

model results with the large quenching rate are incompatible with observations.

\section{Two-Dimensional Model Results}

Figures $2 a, 2 b$, and $2 c$ present the NO distribution calculated by the two-dimensional model as a function of altitude and latitude for low quenching, medium quenching, and high quenching, respectively, of $N\left({ }^{2} D\right)$ by $O$. Recall that the calculations represent solstice, solar cycle minimum conditions during northern summer. For the low-quenching case (Figure 2a), peak NO densities of approximately $1-2 \times 10^{7} \mathrm{~cm}^{-3}$ are found near $110 \mathrm{~km}$ over most of the northern hemisphere. The distribution maximizes in the auroral zone, with densities in the polar night $\geq 10^{8} \mathrm{~cm}^{-3}$. The large nighttime densities in the lower thermosphere and mesosphere at the south pole appear because, at large solar zenith angles, the photochemical sinks of NO are inoperative. Similarly, the low-altitude summer pole exhibits a NO minimum due to strong predissociation in the NO $\delta$ bands. A minimum in the NO latitudinal distribution occurs from about $25^{\circ}$ to $50^{\circ} \mathrm{S}$. In the upper thermosphere, near $200 \mathrm{~km}$, the number densities range from about $5 \times 10^{5}$ to slightly more than $2 \times 10^{6} \mathrm{~cm}^{-3}$ from south to north poles. The intermediate-quenching case (Figure $2 b$ ) has very similar morphology in the upper thermosphere but an appreciable decrease in magnitude of the NO densities near the peak. The peak NO densities are still located near 110 $\mathrm{km}$ for the most part, but now are near $5 \times 10^{6}$ away from the poles. The NO densities near $200 \mathrm{~km}$ are slightly smaller than those calculated with the low quenching rate.

NO densities calculated with the high quenching rate (Figure $2 c$ ) differ dramatically from the previous simulations
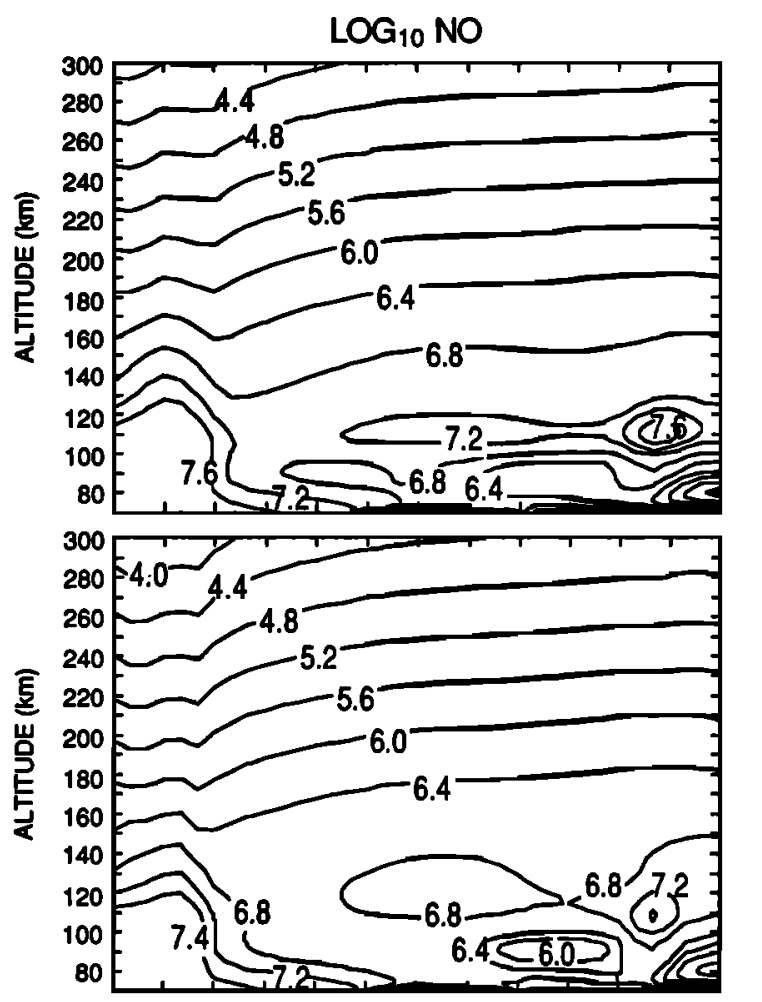

A

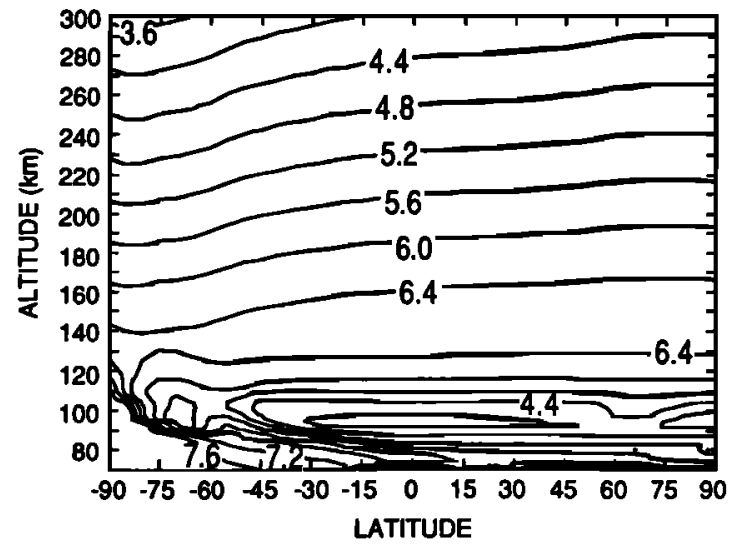

C

Fig. 2. Two-dimensional model calculations of $\log _{10}$ NO with (a) low quenching rate; $(b)$ medium quenching rate : and $(c)$ high quenching rate. Numerical values of rate coefficients are given in Figure 1. 


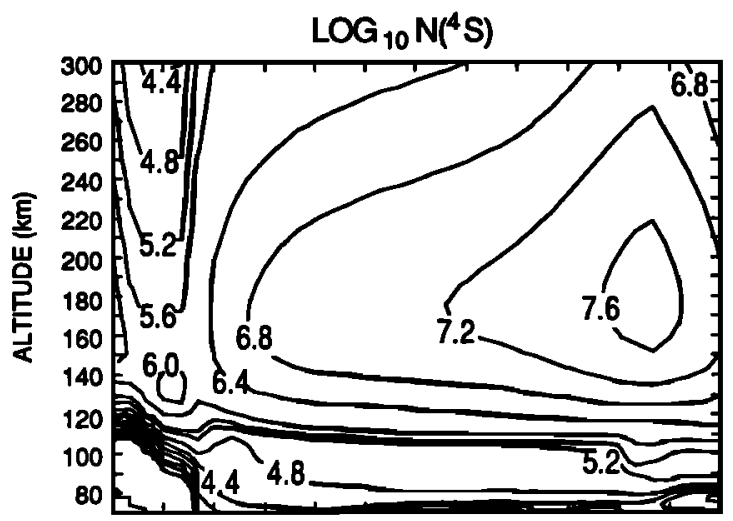

A

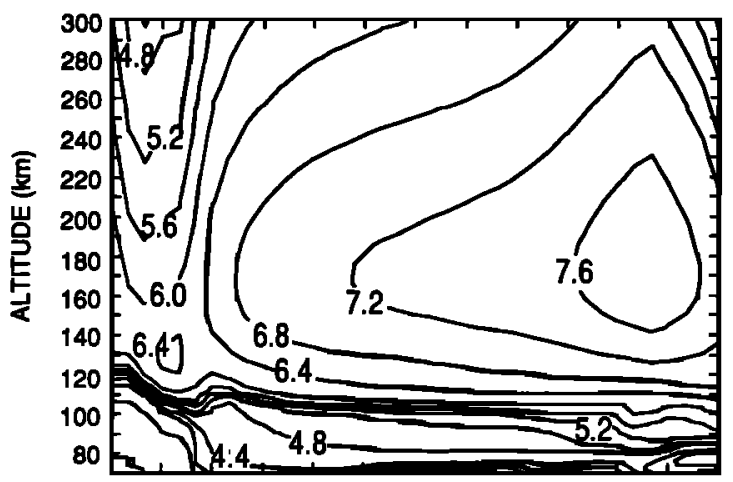

B

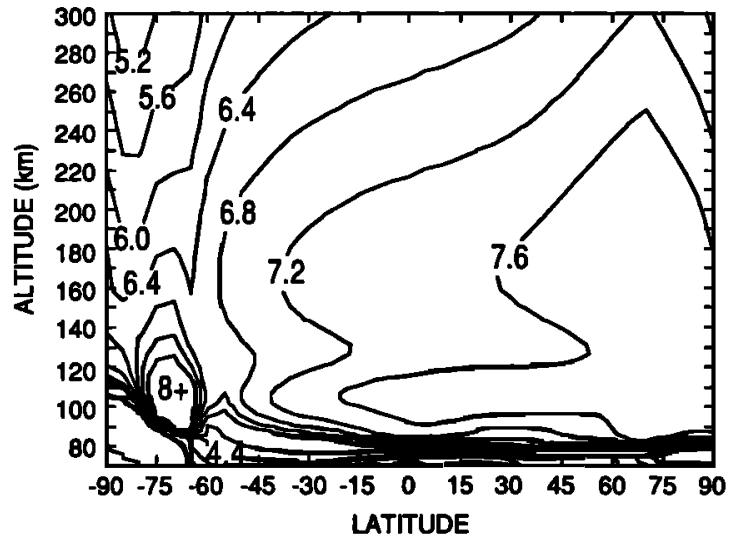

Fig. 3. As Figure 2, but for $\log _{10} N\left({ }^{4} S\right)$ densities.

over the entire altitude range of the model. Peak NO densities of $-3 \times 10^{6}$ now occur near $130-140 \mathrm{~km}$. A deep hole appears in the NO distribution near $90 \mathrm{~km}$, where densities less than $10^{4} \mathrm{~cm}^{-3}$ extend over most latitudes in contradiction with mesospheric rocket observations indicating NO densities greater than $10^{5} \mathrm{~cm}^{-3}$ (see Tohmatsu and Iwagami [1976], Beran and Bangert [1979], and Iwagami and Ogawa [1980]; data reviewed by Solomon et al. [1982]). In the upper thermosphere, near $200 \mathrm{~km}$, the maximum densities are everywhere less than $10^{6} \mathrm{~cm}^{3}$.

The model calculations show that the effect of increasing the quenching rate of $\left.\mathrm{N}^{2} D\right)$ by $\mathrm{O}$ is a decrease in the peak NO densities, accompanied by an increase in the altitude of the peak densities. The different quenching rates will also affect the distributions of $\left.\mathrm{N}^{4} S\right)$ and $\mathrm{N}\left({ }^{2} D\right)$; these are shown in Figures 3 and 4 , respectively. The differences in the $N\left({ }^{4} S\right)$ and $N\left({ }^{2} D\right)$ densities for the low and medium quenching rates (Figures $3 a / 3 b$ and $4 a / 4 b$ ) are confined largely to the lower thermosphere, as expected. A faster $N\left({ }^{2} D\right)$ quenching will de- plete $N\left({ }^{2} D\right)$ and increase $N\left({ }^{4} S\right)$, leading to the reduced NO densities seen in Figure $2 b$. For the large quenching rate, however, the $\mathrm{N}\left({ }^{2} D\right)$ densities (Figure $4 c$ ) are seriously depleted over the entire altitude range of the model: the peak $\mathrm{N}\left({ }^{2} \mathrm{D}\right)$ density is $\sim 6 \times 10^{4} \mathrm{~cm}^{-3}$ near $200 \mathrm{~km}$, compared to 2 $x 10^{6}$ in the previous two simulations. Conversely, the $\mathrm{N}\left({ }^{4} S\right.$ ) densities (Figure $3 c$ ) are greater than $10^{7} \mathrm{~cm}^{-3}$ over much of the model range, with a southern high-latitude pocket of densities greater than $10^{8} \mathrm{~cm}^{-3}$. Figures 1 and 3 show that varying the quenching rate causes dramatic differences in the $\mathrm{N}\left({ }^{4} S\right)$ distribution from about 100 to $160 \mathrm{~km}$. $\mathrm{N}\left({ }^{4} S\right)$ measurements from 100 to $200 \mathrm{~km}$ were presented by $M c C o y[1983 a, b]$, which show a rapid decrease in $N\left({ }^{4} S\right)$ below about $140 \mathrm{~km}$, with the densities dropping from $\sim 10^{7}$ to $10^{5}$ or less at $100 \mathrm{~km}$. The model results with the large quenching rate (Figures $1 c$ and $3 c$ ) are not consistent with the McCoy observations.

Figure 5 compares the latitudinal distribution of nitric oxide measured by the ultraviolet spectrometer on board the

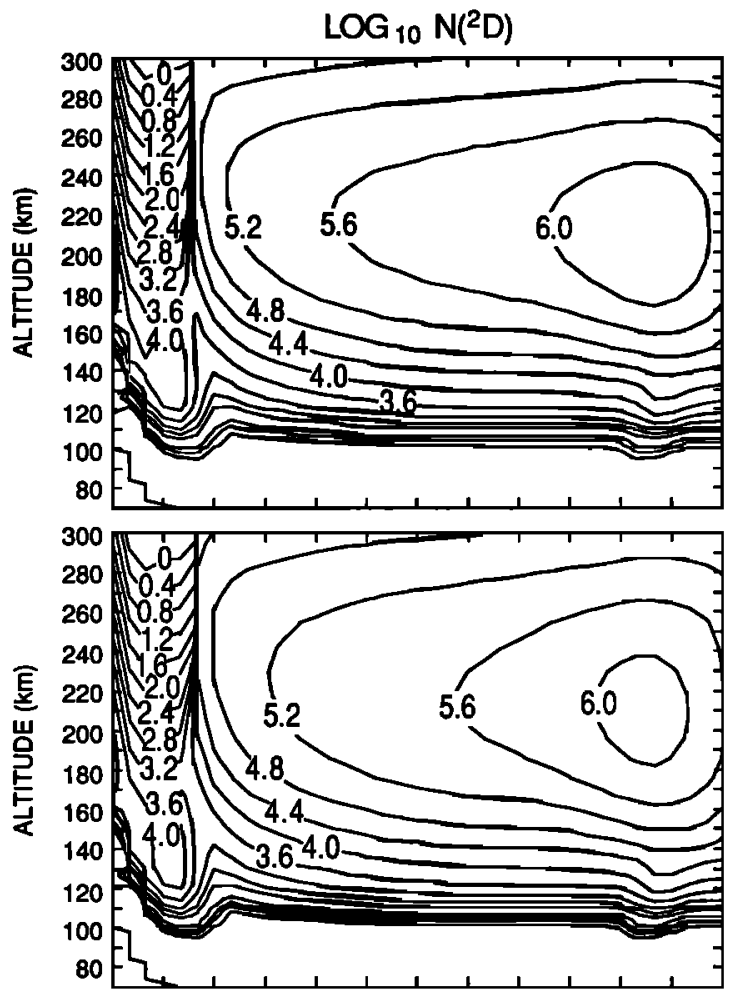

A

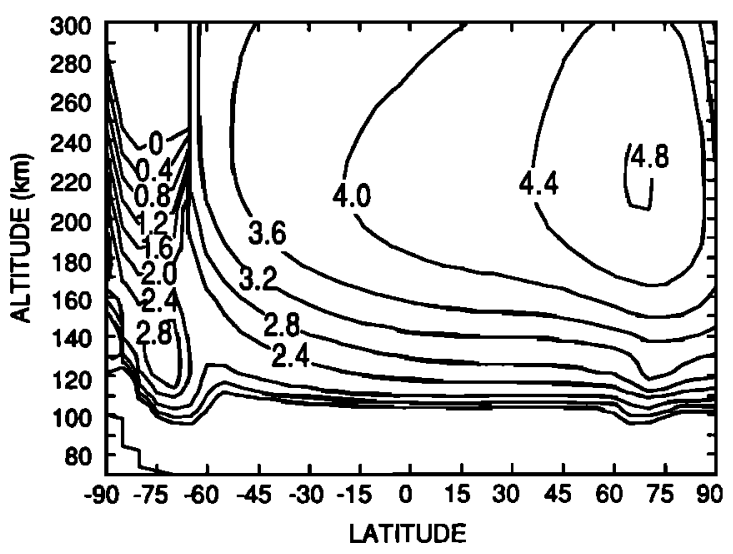

C

Fig. 4. As Figure 2, but for $\log _{10} \mathrm{~N}\left({ }^{2} D\right)$ densities. 


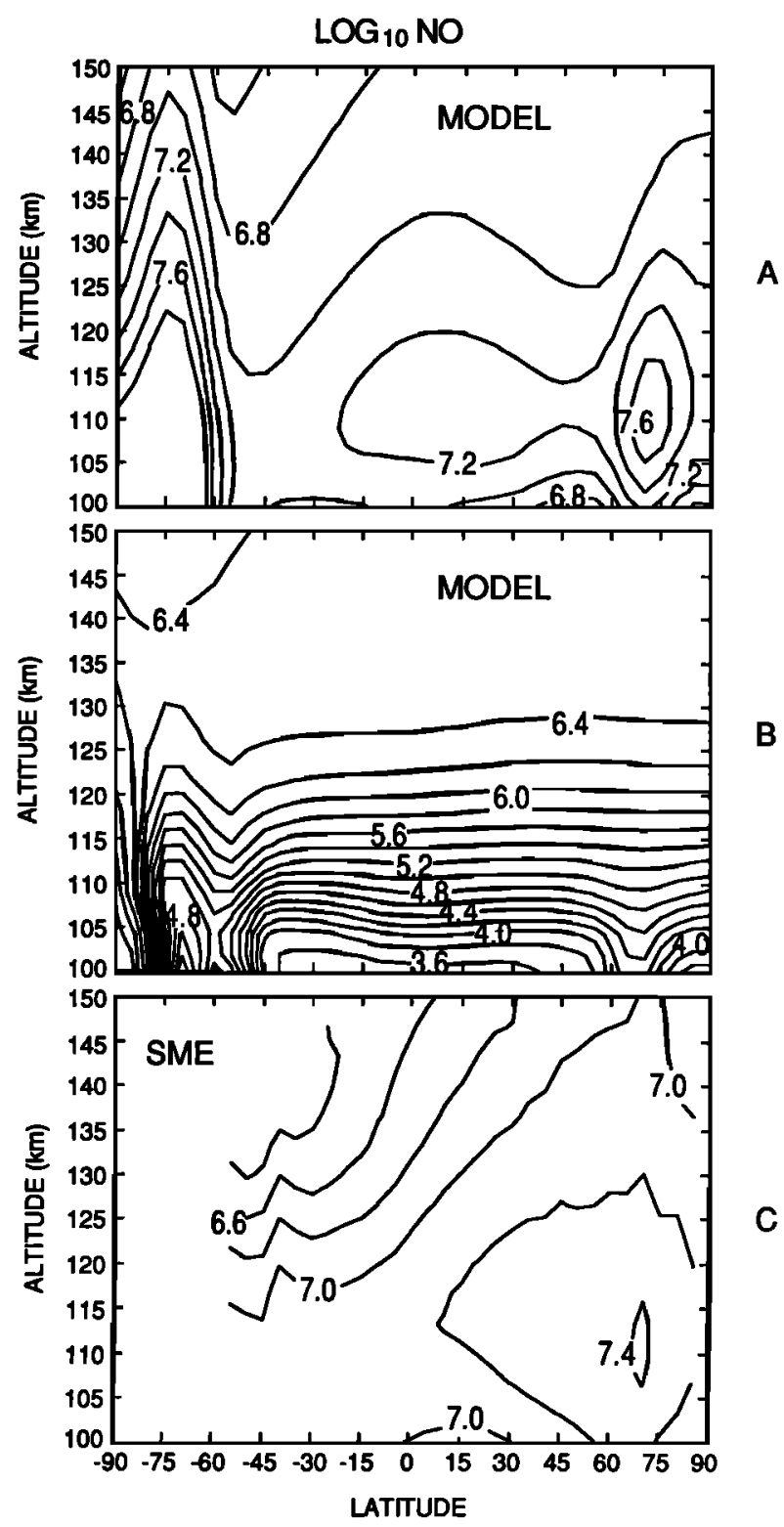

Fig. 5. Comparison of $\log _{10}$ NO densities as calculated by the twodimensional model and observed by the SME satellite. (a) two-dimensional model calculations with low quenching rate, (b) two-dimensional model calculations with fast quenching rate, and (c) SME observations averaged from June 5 through July 5, 1984.

Solar Mesosphere Explorer satellite [Rusch et al., 1984; Barth, 1988] during a solstice period in 1984 (Figure 5c) with the results of the two-dimensional model with the low (Figure $5 a$ ) and high (Figure $5 b$ ) quenching rates, respectively. The SME data were obtained during the period June 5 through July 5, 1984, comprising approximately 30 orbits. The SME is in a nearly polar circular orbit at $520 \mathrm{~km}$, obtaining data near $1500 \mathrm{LT}$. The observations used to produce this map were gathered in the European ( $45^{\circ} \mathrm{E}$ longitude) and American $\left(120^{\circ} \mathrm{W}\right.$ longitude) sectors. The mean $A p$ magnetic index value was 14 , corresponding to a fairly magnetically quiet period, while the average solar $F 10.7$ flux was $\sim 100$.

The contours of NO density are plotted as a function of geomagnetic latitude as suggested by Gérard and Nöel [1986]. This method improves the organization of the NO meridional distribution, particularly at high latitudes. The NO distribu- tion obtained with the two-dimensional model and shown in Figure $2 a$ and $2 c$ is plotted here on the same scale as the SME observations to facilitate comparisons. Although the model was not "tuned" to match exactly the geophysical conditions of the SME measurements, the main features of the observations are satisfactorily reproduced in Figure $5 a$ (low $\mathrm{N}\left({ }^{2} D\right)$ quenching coefficient). The NO peak altitude calculated with the model is located near $110 \mathrm{~km}$, in good agreement with the SME observations. As with the one-dimensional model results, the calculations done with the fast quenching rate produce NO densities that are clearly inconsistent with the observations.

The position of the latitudinal minimum in the two-dimensional model results for low and medium quenching rates is about $30^{\circ} \mathrm{N}$ of its observed location. Gérard et al. [1984] showed that this is a function of auroral activity: an increase in the Joule heating and particle precipitation, as occurs during disturbed periods, will shift the minimum to lower latitudes. The model results shown here represent quiet geomagnetic conditions, while the data are averaged over many days covering both disturbed and quiescent periods. Further, the model represents a diumal average variation of NO, while the AE observations are restricted to daylight hours. It is therefore not appropriate to attempt an exact reproduction of the satellite observations; their usefulness in this study is to illustrate trends rather than absolute values.

\section{Discusston}

Satellite measurements of odd-nitrogen densities are very difficult to reconcile with theoretical models if the quenching rate of $\mathrm{N}\left({ }^{2} D\right)$ by $\mathrm{O}$ is as large as measured by Jusinski and colleagues [Jusinski and Slanger, 1987; Jusinski et al., 1988]. Even if the upper thermosphere NO and $N\left({ }^{4} S\right)$ densities could be reconciled with the fast quenching rate by adjusting various reaction or branching rates, the low-altitude NO densities would still likely remain seriously underestimated. Use of the fast quenching rate in the model calculations cannot simultaneously satisfy both lower and upper thermosphere measurements. In the upper thermosphere, $N\left({ }^{4} S\right)$ is both a source and a sink of NO. Rapid quenching of $\mathrm{N}\left({ }^{2} D\right)$ by $\mathrm{O}$ would eliminate $\mathrm{N}\left({ }^{2} D\right)$ as a source of NO, while increasing the $\mathrm{N}\left({ }^{4} S\right)$ concentration. In the lower thermosphere, $\mathrm{N}\left({ }^{4} S\right)$ is a sink of NO, while $\mathrm{N}\left({ }^{2} D\right)$ is a major source. $A$ very fast $\mathrm{N}\left({ }^{2} D\right)$ quenching rate therefore implies both an accelerated loss of NO coupled with a greatly reduced production. Thus, Figure $1 c$ shows $100-\mathrm{km}$ NO densities near $10^{5}$ $\mathrm{cm}^{-3}$ which are incompatible with every measurement known near this altitude. Figure $1 c$ also shows that the $N\left({ }^{2} D\right)$ model simulations are completely unacceptable in comparison with the satellite observations. The VAE instrument on the AE satellite routinely measured the $520.0-\mathrm{nm}$ emission from $\mathrm{N}\left({ }^{2} D\right)$, which would be far below the detection limit of the instrument if the quenching rate were as large as Jusinski and colleagues suggest. The existence of "hot" $\mathrm{N}$ atoms could possibly augment the NO densities in the high-quenching case, but the problem of the very low $\mathrm{N}\left({ }^{2} D\right)$ densities would remain.

The resolution of this problem requires two efforts. Additional, independent laboratory measurements of the $\mathrm{N}\left({ }^{2} D\right)+$ $O$ quenching rate are clearly necessary. At the same time, there is only one set of simultaneous measurements of NO, 
$\mathrm{N}\left({ }^{4} S\right)$, and $\mathrm{N}\left({ }^{2} D\right)$ for model comparisons. Additional simultaneous data on all three constituents are required to validate the conclusions drawn here.

Acknowledgments. The SME satellite data were kindly provided by Charles A. Barth. We are grateful to the two referees for helpful comments. This work was supported by NASA grant NAGW-904 to the University of Colorado and NATO Co-operative Research Grant 166/84. J.-C. G. also acknowledges the support of the Belgian Foundation for Scientific Research (FNRS). The National Center for Atmospheric Research provided computer time for this project. NCAR is sponsored by the National Science Foundation.

The Editor thanks R. P. McCoy and T. G. Slanger for their assistance in evaluating this paper.

\section{REFERENCES}

Barth, C. A., Rocket measurements of the nitric oxide dayglow, $J$. Geophys. Res., 69, 3301, 1964.

Barth, C. A., Reference models for thermospheric NO, paper presented at COSPAR Meeting, Helsinki, Finland, 1988.

Barth, C. A., D. W. Rusch, and A. I. F. Stewart, The UV nitric-oxide experiment for Atmosphere Explorer, Radio Sci., 8, 379, 1973.

Becker, K. H., W. Groth, and D. Z. Kley, Z. Naturforschu A, 24, 1280, 1969.

Beran, D., and W. Bangert, Trace constituents in the mesosphere and lower thermosphere during winter anomaly events, J. Atmos. Terr. Phys., 41, $1091,1979$.

Cogger, L. L., J. C. G. Walker, J. W. Meriwether, and R. G. Bumside, $F$ region airglow: Are groundbased observations consistent with recent satellite results?, J. Geophys. Res., 85, 3013, 1980.

Cravens, T. E., and T. L. Killeen, Longitudinally asymmetric transport of nitric oxide in the $E$ region, Planet. Space Sci., 36, 11, 1988.

Cravens, T. E., and A. I. F. Stewart, Global morphology of nitric oxide in the lower $E$ region, J. Geophys. Res., 83, 2446, 1978.

Cravens, T. E., J.-C. Gérard, M. LeCompte, A. I. F. Stewart, and D. W. Rusch, The global distribution of nitric oxide in the thermosphere as determined by the Atmosphere Explorer D satellite, J. Geophys. Res., 90, 9862, 1985.

Dalgarno, A., W. B. Hanson, N. W. Spencer, and E. R. Schmerling, The Atmosphere Explorer mission, Radio Sci., 8, 263, 1973.

Davenport, J. E., T. G. Slanger, and G. Black, The quenching of $\mathrm{N}\left({ }^{2} D\right)$ by $O\left({ }^{3} P\right), J$. Geophys. Res., 81, $12,1976$.

Ebel, A., Eddy diffusion models for the mesosphere and lower thermosphere, J. Atmos. Terr. Phys., 42, 617, 1980.

Frederick, J. E., and D. W. Rusch, On the chemistry of metastable atomic nitrogen in the $F$ region deduced from simultaneous satellite measurements of the $5200-\AA$ airglow and atmospheric composition, J. Geophys. Res., 82, 3509, 1977.

Garstang, R. H., Transition probabilities in auroral lines, in The Airglow and Aurore, edited by E. B. Amstrong and A. Dalgamo, p. 324, Pergamon, New York, 1956.

Gérard, J.-C., and C. E. Nöel, AE-D measurements of the NO geomagnetic latitudinal distribution and contamination by $\mathrm{N}^{+}\left({ }^{5} S\right)$ emission, J. Geophys. Res., 91, 10136, 1986.

Gerrard, J.-C., and R. G. Roble, The role of nitric oxide on the zonally averaged structure of the thermosphere: Solstice conditions for solar cycle minimum, Planet. Space Sci., 34, 131, 1986.

Gérard, J.-C., and R. G. Roble, The role of nitric oxide on the zonally averaged structure of the thermosphere: Solstice conditions for solar cycle maximum, Planet. Space Sci, 36, 271, 1988.

Gerard, J.-C., and C. Taieb, The $E$ region electron density diumal asymmetry at Saint-Santin: Observations and role of nitric oxide, J. Atmos. Terr. Phys., 48, 471, 1986.
Gérard, J.-C., R. G. Roble, D. W. Rusch, and A. I. F. Stewart, The global distribution of thermospheric odd nitrogen for solstice conditions during solar cycle minimum, J. Geophys. Res., 89, $1725,1984$.

Godefroid, M., and C. Froese Fischer, MCHF-BP fine structure splittings and transition rates for the ground configuration in the nitrogen sequence, J. Phys. B, 17, 681, 1984.

Hays, P. B., G. Carignan, B. C. Kennedy, G. G. Shepherd, and J. C. G. Walker, The Visual Airglow Experiment on Atmosphere Explorer, Radio Sci., 8, 369, 1973.

Iannuzzi, M. P., and F. Kaufman, Rates of some reactions of $\mathrm{N}^{2} D$ and ${ }^{2} P$ ) near $300 \mathrm{~K}, J$. Chem. Phys., 73, 4701, 1980.

Iwagami, N., and T. Ogawa, An antarctic NO density profile deduced from the gamma band airglow, Planet. Space Sci., 28, 867. 1980.

Iwagami, N., and T. Ogawa, Thermospheric NO profiles observed at the diminishing phase of solar cycle 21, Planet. Space Sci., 35, 191, 1987.

Jusinski, L. E., and T. G. Slanger, Determination of rate coefficient for quenching $\mathrm{N}\left({ }^{2} D\right)$ by $O\left({ }^{3} P\right)$, Eos Trans. AGU, 68, 1389, 1987.

Jusinski, L. E., G. Black, and T. G. Slanger, REMPI measurements of $\mathrm{N}\left({ }^{2} D\right)$ quenching by $\mathrm{O}\left({ }^{3} P\right), J$. Phys. Chem., 92, 5977, 1988.

Kley, D., G. M. Lawrence, and E. J. Stone, The yield of $\left.\mathrm{N}^{2} D\right)$ atoms in the dissociative recombination of $\mathrm{NO}^{+}, \mathrm{J}$. Chem. Phys., 66, 4157, 1977.

Lee, J. H., J. V. Michael, W. A. Payne, and L. J. Stief, Absolute rate of the creation of $\left.\mathrm{N}^{4} \mathrm{~S}\right)$ with NO from $196-400^{\circ} \mathrm{K}$ with DF-RAF and FP-RF techniques, J. Chem. Phys., 69, 3069, 1978.

Lin, C.-L., and F. Kaufman, Reactions of metastable nitrogen atoms, J. Chem. Phys., 55, 3760, 1971.

McCoy, R. P., Thermospheric odd nitrogen, 1, NO, N $\left({ }^{4} S\right)$, and $\left.O{ }^{3} P\right)$ densities from rocket measurements of the NO $\delta$ and $\gamma$ bands and the $\mathrm{O}_{2}$ Herzberg I bands, $J$. Geophys. Res., 88, 3197, $1983 a$.

McCoy, R. P., Thermospheric odd nitrogen, 2, Comparison of rocket observations with a diffusive transport chemical model, $J$. Geophys. Res., 88, 3206, $1983 b$.

McFarland, M., D. L. Albrituon, F. C. Fehsenfeld, E. E. Ferguson, and A. L. Schmeltekopf, Energy dependence and branching ratio of the $\mathrm{N}_{2}{ }^{+}+\mathrm{O}$ reaction, J. Geophys. Res., 79, 2925, 1974.

Nicolet, M., and A. C. Aikin, The formation of the $D$ region of the ionosphere, J. Geophys. Res., 65, 1469, 1960.

Norton, R. B., and C. A. Barth, Theory of nitric oxide in the Earth's atmosphere, J. Geophys. Res., 75. 3903, 1970.

Ogawa, T., N. Iwagami, and Y. Kondo, Solar cycle variation of thermospheric nitric oxide, J. Geomagn. Geoelectr., 36, 317, 1984.

Richards, P. G., and D. G. Torr, Ratio of photoelectron to EUV ionization rates for aeronomic studies, J. Geophys. Res., 93, 4060, 1988.

Richards, P. G., D. G. Torr, and M. R. Torr, Photodissociation of $\mathrm{N}_{2}$ : A significant source of thermospheric atomic nitrogen, $J$. Geophys. Res., 86, 1495, 1981.

Ridley, E. C., R. G. Roble, and R. E. Dickinson, Global distribution of atomic nitrogen and nitric oxide calculated by the NCAR thermospheric general circulation model, Eos Trans. AGU, 68, 1388, 1987.

Roble, R. G., and B. A. Emery, On the global mean temperature of the thermosphere, Planet. Space Sci., 31, 597, 1983.

Roble, R. G., and J. F. Kasting, The zonally averaged circulation, temperature, and compositional structure of the thermosphere and variations with geomagnetic activity, J. Geophys. Res., 89, $1711,1984$.

Rusch, D. W., Satellite ultraviolet measurements of nitric oxide 
fluorescence with a diffusive tnansport model, J. Geophys. Res., 78, 5676, 1973.

Rusch, D. W., and J.-C. Gérard, Satellite studies of $\mathrm{N}\left({ }^{2} D\right)$ emission and ion chemistry in aurorae, J. Geophys. Res., 85, 1285, 1980.

Rusch, D. W., A. I. F. Stewart, P. B. Hays, and J. H. Hoffman, The N I (5200 A) dayglow, J. Geophys. Res., 80, 2300, 1975.

Rusch, D. W., G. H. Mount, C. A. Barth, R. J. Thomas, and M. T. Callan, Solar Mesosphere Explorer ultraviolet spectrometer: Measurements of ozone in the 1.0-0.1 mbar region, J. Geophys. Res., 89, 11677, 1984.

Solomon, S., The possible effects of translationally excited nitrogen atoms on lower thermospheric odd nitrogen, Planet. Space Sci., $31,135,1983$.

Solomon, S., and R. R. Garcia, Transpont of thermospheric NO to the upper stratosphere, Planet. Space Sci., 32, 399, 1984.

Solomon, S., R. G. Roble, and P. J. Crutzen, Photochemical coupling between the thermosphere and the lower atmosphere, 1, Odd nitrogen from 50 to $120 \mathrm{~km}, J$. Geophys. Res., 87, 7206, 1982.

Spencer, N. W., H. B. Neimann, and G. R. Carignan, The neutral atmosphere temperature instrument, Radia Sci., 8, 284, 1973.

Stewar, A. I. F, and T. E. Cravens, Diumal and seasonal effects in $E$ region low-latitude nitric oxide, J. Geophys. Res., 83, 2453, 1978.

Strickland, D. J., and R. R. Meier, A photoelectron model for the rapid computation of atmospheric excitation rates, NRL Memo. Rep., 5004, 1982.

Strobel, D. F., D. N. Hunten, and M. B. McElroy, Production and diffusion of nitric oxide, J. Geophys. Res., 75, 4307, 1970.

Swider, W., Daytime nitric oxide at the base of the thermosphere, $J$. Geophys. Res., 83, 4407, 1978.

Tohmatsu, T., and N. Iwagami, Measurement of nitric oxide abundance in the equatorial upper atmosphere, J. Geomagn. Geoelectr., 28, 343, 1976.

Torr, D. G., J. C. G. Walker, L. H. Brace, J. H. Hoffmann, A. O. Nier, and $\mathrm{M}$. Oppenheimer, Recombination of $\mathrm{NO}^{+}$in the ionosphere, Geophys. Res. Lett., 3, 209, 1976.

Torr, M. R., and D. G. Torr, Ionization frequencies for solar cycle 21: Revised, J. Geophys. Res., 90, 6675, 1985.

Zeippen, C. J., Transition probabilities for forbidden lines in the $2 p^{3}$ configuration, Mon. Not. R. Astron. Soc., 198, 111, 1982.

C. G. Fesen and D. W. Rusch, Laboratory for Atmospheric and Space Physics, University of Colorado, Campus Box 392, Boulder, CO 80309-0392.

J.-C. Gérard, Institut d'Astrophysique, Université de Liège, 4200 Liège-Ougrée, Belgium.

(Received July 12, 1988; revised November 28, 1988; accepted December 1, 1988) 